\title{
El rol de la Hidrología Subterránea para el logro de la Seguridad Hídrica
}

\section{The role of Hydrogeology to achieve the Water Security}

\author{
PARIS M. ${ }^{1}$, PÉREZ M. ${ }^{2}$ y D'ELÍA M. ${ }^{3}$ \\ 1 Facultad de Ingeniería y Ciencias Hídricas. Universidad Nacional del Litoral (FICH-UNL). \\ parismarta@gmail.com \\ ${ }^{2}$ Facultad de Ingeniería y Ciencias Hídricas. Universidad Nacional del Litoral (FICH-UNL). \\ maperez@fich.unl.edu.ar \\ ${ }^{3}$ Facultad de Ingeniería y Ciencias Hídricas. Universidad Nacional del Litoral (FICH-UNL). \\ mdelia@fich.unl.edu.ar
}

\section{Resumen}

El concepto de seguridad hídrica focaliza el objetivo de la gestión del agua en la sociedad. Sin embargo, en los ambientes urbanos -donde deben atenderse requerimientos relacionados al abastecimiento de agua, inundaciones, lluvias intensas, ascenso del nivel freático, problemas de contaminación, entre otros-, la falta de datos e información adecuadas a la escala de trabajo, e incluso el uso inapropiado de metodologías, atentan contra cualquier intento de mejorar esta capacidad de la población para hacer frente a las situaciones críticas. Incluso 
en las ciudades es más evidente que el desafío de lograr el equilibrio entre la oferta de agua -finita, vulnerable y variable-, y una oferta -incansablemente creciente, de múltiples usuarios e interdependiente-, obliga a "re pensar" el ciclo hidrológico como un sistema ambiental dinámico y con ello, a sumar a la Hidrología un enfoque interdisciplinar. En este trabajo se presentan las metodologías, resultados y conclusiones que han servido de base para mejorar el conocimiento de los sistemas acuíferos de las ciudades de Esperanza y Santa Fe (Argentina). La primera sustenta su abastecimiento con aguas subterráneas, con lo cual es indispensable la protección del recurso y las obras de captación. En el segundo caso, la interrelación entre el agua superficial y la subterránea es compleja y requiere que hidrodinámica del sistema en su conjunto deba ser considerada para definir las mejores alternativas tecnológicas de la infraestructura urbana y el manejo de las inundaciones. Los avances logrados señalan la importancia de la ciencia, como motor que genera soluciones efectivas para que la sociedad alcance las metas del desarrollo sostenible y la seguridad hídrica.

Palabras clave: Hidrogeología, Aguas subterráneas, Argentina, Gestión, Seguridad Hídrica.

\section{ABSTRACT}

The concept of water security focuses on the objective of water management in society. However, in urban environments -where requirements related to water supply, floods, important rains, rising water tables, pollution problems, among others-, the lack of data and information appropriate to the scale of work, and even the inappropriate use of methodologies, attempt against any attempt to improve this capacity of the population to deal with critical situations. Even in the cities, it is more evident that the challenge of achieving a balance between the supply of water -finite, vulnerable and variable-, and an offer - tirelessly growing, of multiple users and interdependent-, forces us to "rethink" the hydrological cycle as a dynamic environmental system and with it, to add an interdisciplinary approach to hydrology. This paper presents the methodologies, results and conclusions that have served as the basis for improving knowledge of aquifer systems in the cities of Esperanza and Santa Fe (Argentina). The first supports its supply with groundwater, which is essential to protect the resource and the collection works. In the second case, the interrelation between surface and underground water is complex and requires that hydrodynamics of the system as a whole should be considered to define the best technological alternatives for urban infrastructure and the management of floods. The advances achieved point to the importance of science, as an engine that generates effective solutions for society to achieve the goals of sustainable development and water security.

Keywords: Hydrogeology, Groundwater, Argentina, Management, Water Security. 


\section{INTRODUCCIÓN}

El aumento de la demanda de agua, la variabilidad y cambio climático, el deterioro de la calidad por contaminación, la dispar distribución espacio-temporal de los recursos hídricos y la falta o fallas en la gobernanza son algunas de las causas usualmente mencionadas para explicar la actual crisis hídrica. Efectivamente, no puede negarse que desde un tiempo a esta parte hay cambios con consecuencias importantes relacionados con el agua.

La demanda mundial aumenta a un ritmo del $1 \%$ en función de diversos factores como el aumento de la población, los cambios en los patrones de consumo, las necesidades de agua para producir alimentos y energía, etc. Se estima incluso que la demanda industrial y la doméstica crecerán más rápidamente que la agrícola, aunque esta última seguirá siendo el mayor consumidor de agua. Por otra parte, en lo que respecta a la oferta de agua, el cambio climático global lleva a que las regiones más húmedas sean cada vez más húmedas, las más secas aún más secas y en general se agraven los eventos hidrometerológicos extremos como lluvias intensas que conducen a crecidas, inundaciones, derrumbes, deslizamientos, etc. El aumento en la carga de nutrientes por la aplicación no controlada de agroquímicos, la presencia de microorganismos patógenos, consecuencia de la falta de obras de saneamiento, la inadecuada o ausente gestión de residuos y efluentes (domiciliarios, industriales, rurales, mineros, etc.), son causas importantes del deterioro de las reservas de agua (superficial y subterránea) y de la afectación de la salud humana y de los ecosistemas (WWAP/ONU, 2018).
En los ambientes urbanos -donde deben atenderse requerimientos relacionados al abastecimiento de agua, inundaciones, lluvias intensas, ascenso del nivel freático, problemas de contaminación, entre otros-, la falta de datos e información adecuadas a la escala de trabajo, e incluso el uso inapropiado de metodologías, atentan contra cualquier intento de mejorar la seguridad de la población para hacer frente a las situaciones críticas. Incluso en las ciudades es más evidente que el desafío de lograr el equilibrio entre la oferta de agua -finita, vulnerable y variable-, y una oferta -incansablemente creciente, de múltiples usuarios e interdependiente-, que obliga a "re pensar" el ciclo hidrológico como un sistema ambiental dinámico y con ello, a sumar a la Hidrología un enfoque interdisciplinar.

El objetivo de esta ponencia es mostrar el rol fundamental del conocimiento de los sistemas hidrológicos en la gestión del agua, cuando los mismos son logrados a partir de un enfoque integrado que considere la multidimensionalidad de los problemas hídricos.

\section{MARCO TEÓRICO}

El concepto de seguridad hídrica comenzó a instalarse en el II Foro Mundial del Agua (La Haya, 2000) y desde ese momento ha ido evolucionando. Sin dudas el punto clave es entender que orienta la definición de las políticas públicas en el tema agua, define criterios para establecer metas y aporta indicadores para evaluar la efectividad de las decisiones. Todo ello adaptado a cada realidad, para cada contexto, para cada comunidad, pues describe una característica de la población. Además, plantea el desafío de aceptar la existencia del riesgo y señala la necesidad de contar con capacidades ins- 
titucionales, financieras y de infraestructura para minimizar la "inseguridad" (Shah, 2016) (Van Beek, Lincklaen Arriens, 2014).

UNESCO (2014) muestra define de una manera clara y amplia a la seguridad hídrica como "la capacidad de una población para salvaguardar el acceso a cantidades adecuadas de agua de calidad aceptable, que permita sustentar tanto la salud humana como la del ecosistema, basándose en las cuencas hidrográficas, así como garantizar la protección de la vida y la propiedad contra riesgos relacionados con el agua -inundaciones, derrumbes, subsidencia de suelos y sequías"

De acuerdo con Peña (2016) la seguridad hídrica consiste en tener:

- Una disponibilidad de agua que sea adecuada, en cantidad y calidad, para el abastecimiento humano, los usos de susbsistencia, la protección de los ecosistemas y la producción.

- La capacidad -institucional, financiera y de infraestructura- para acceder y aprovechar dichos recursos de forma sustentable y manejar las interrelaciones y externalidades entre los diferentes usos y sectores, de manera coherente.

- Un nivel aceptable de riesgos para la población, el medio ambiente y la economía, asociados a los recursos hídricos.

Esto significa que el sujeto de la seguridad hídrica es la población, que los aspectos organizacionales de la sociedad son un factor determinante y que la seguridad absoluta no existe, debe asumirse la incertidumbre asociada a los procesos naturales y procurar los medios para mejorar minimizar la inseguridad.

El concepto permite traducir las metas globales - plasmadas en los Objetivos del Desarrollo Sostenible (ODS) enunciados en la Agenda 2030 (PNUD, 2015)- al nivel local. Así, mientras la seguridad hídrica indica qué se debe tener para lograr alcanzar el desarrollo sostenible, y la gestión integrada de los recursos hídricos (GIRH) es el vehículo para lograrlo. Se trata de un enfoque que resulta ser el catalizador para la articulación de distintas disciplinas que confluyen y aportan la indiscutiblemente necesaria base del conocimiento para entender los problemas del agua en sus múltiples dimensiones.

\section{CASOS DE ESTUDIO: METODOLOGÍA Y RESULTADOS.}

En este trabajo se presentan las metodologías y resultados que han servido de base para mejorar el conocimiento de los sistemas hídricos de las ciudades de Esperanza y Santa Fe (Argentina). La primera sustenta su abastecimiento con aguas subterráneas, con lo cual es indispensable garantizar la protección del recurso (en cantidad y calidad), las obras de captación y proponer un modelo de gestión para el abastecimiento sostenible del agua. En el segundo caso, la compleja interrelación entre el agua superficial y la subterránea requiere que hidrodinámica del sistema en su conjunto deba ser considerada en su conjunto para prevenir y manejar el ascenso del nivel freático y su relación con la infraestructura urbana, el control de la contaminación y las inundaciones/anegamiento. Como se deduce, ambas situaciones refieren a la seguridad hídrica de la población.

\section{Protección de las fuentes de abastecimiento de agua en Esperanza (Argentina)}

El servicio de agua potable por red en la ciudad de Esperanza data del año 1930. Por aquel entonces Obras Sanitarias de la Na- 
ción (OSN) construyó una red de distribución alimentada por 3 pozos que producían alrededor de $180 \mathrm{~m}^{3} / \mathrm{h}$. Hacia fines del año 1994, el servicio sanitario (como en otras localidades de la provincia) estuvo a cargo de una empresa privada concesionaria (Aguas Provinciales de Santa Fe Sociedad Anónima - APSF). Hasta aproximadamente el año 1997 esta empresa concesionaria llevó adelante un esquema de explotación intensiva del acuífero. La creciente demanda, los peligros de contaminación asociados a la actividad económica y la falta de obras de saneamiento, condujeron al continuo deterioro de la fuente de abastecimiento.

Desde 1997 la ciudad de Esperanza es objeto de estudios encarados por la Facultad de Ingeniería y Ciencias Hídricas (Universidad Nacional del Litoral) (FICH-UNL). Los resultados obtenidos permitieron definir en el año 1999 un modelo de gestión para garantizar el uso sostenible de los recursos hídricos disponibles en el área y acompañar el desarrollo sostenible de la región. Se determinaron el diseño y distancia entre las obras de captación como también su esquema de explotación (caudal máximo, horas de funcionamiento) y diseño de los pozos de monitoreo del sistema acuífero mediante la implementación de un modelo matemático hidrogeológico desarrollado en Visual Modflow. Los escenarios de explotación ensayados dieron lugar a diferentes alternativas de gestión para 20 años de proyección de la demanda.

A fin de dar respuesta a la creciente demanda de agua de ambas ciudades, con- siderar el peligro de contaminación de las fuentes de abastecimiento (pozos) por la actividad industrial y de servicios que se desarrolla en el ejido urbano, el deterioro de la calidad del agua subterránea por ascenso de agua salina, y la búsqueda de consenso entre los distintos estamentos de ambas comunidades (empresa prestadora, ONGs, gobiernos locales y provincial, ente regulador, profesionales, técnicos, científicos, etc.) el modelo de gestión contempló algunas medidas como:

- reubicar los pozos en la zona rural de Esperanza, en un campo de bombeo único para las dos localidades, fuera de la influencia del área urbana.

- aumentar el número de perforaciones respetando un espaciamiento entre las mismas no menor a $500 \mathrm{~m}$

- reducir los caudales unitarios de extracción a una tasa de $1200 \mathrm{~m}^{3} /$ día a razón de 20 horas diarias de funcionamiento máximo (caudal promedio de $\left.60 \mathrm{~m}^{3} / \mathrm{h}\right)$.

Esta propuesta condujo al diseño del actual campo de bombeo, localizado hacia el oeste de la ciudad, principalmente en zona rural (Figura 1). El mismo está compuesto por 32 pozos que abastecen además a la localidad de Rafaela (unos $50 \mathrm{~km}$ al oeste de Esperanza), a cargo desde el año 2004 de la Empresa Aguas Santafesinas S.A. (sociedad anónima del Estado Provincial). El único tratamiento que recibe es el de cloración, con hipoclorito de sodio, previo a su distribución que abarca el 92\% del ejido urbano de Esperanza. 

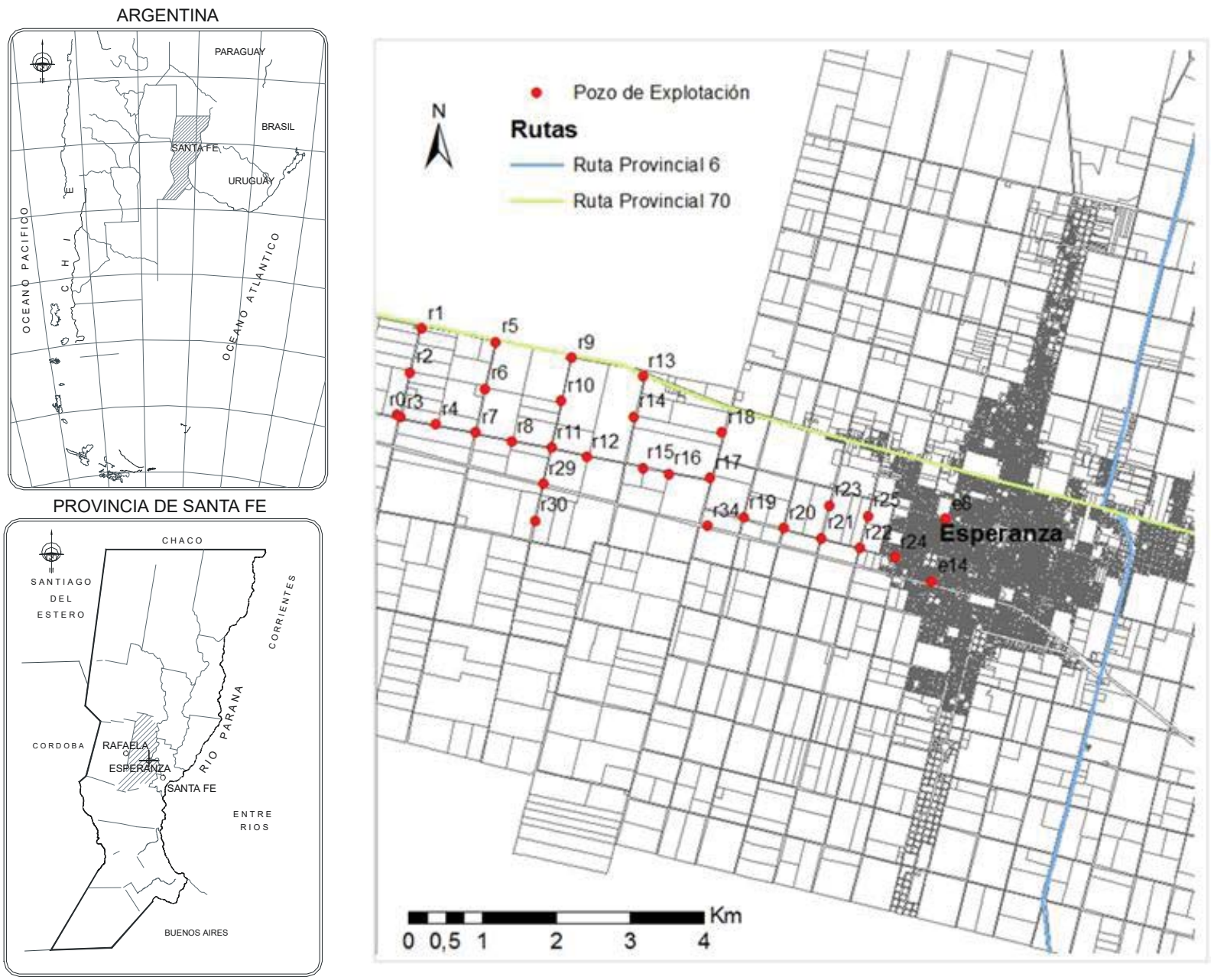

Fig. 1. Ubicación de las obras de captación en el campo de bombeo de la ciudad de Esperanza.

Este modelo de gestión se basó en la sólida construcción del conocimiento de la hidrogeología, hidrodinámica e hidroquímica del sistema acuífero. Sobre la base de conceptualizaciones antecedentes (Filí, Tujchneider, Paris, Pérez, D’Elía, 1999) (Tujchneider, Filí, Paris, D’Elía, Pérez, 1998) y la interpretación de perfiles litológicos y estudios disponibles, se ha elaborado el esquema del modelo conceptual de funcionamiento del sistema hidrogeológico en el área de estudio (Figura 2).

El estrato superior, con un espesor variable entre 15 y $25 \mathrm{~m}$, corresponde al Grupo Pampa (sedimentos pampeanos) (Pleistoce- no lacustre-palustre-eólico). Se trata de limos, arcillas y loess, pardos claros, oscuros, rojizos o grises, según el ambiente de depositación, con presencia de tosca en algunos niveles. Aloja un acuífero libre de bajo rendimiento y calidad variable que se explota fundamentalmente para las actividades agrícolas. En la porción más profunda, donde los sedimentos son más arcillosos (2-3m de espesor promedio) se comportan como acuitardo. Inferiormente se localiza la Formación Ituzaingó (arenas puelches) (Plioceno fluvial) compuesta por arenas finas, medianas y gruesas amarillas, con recubrimiento limonítico. Aloja un acuífero semiconfina- 


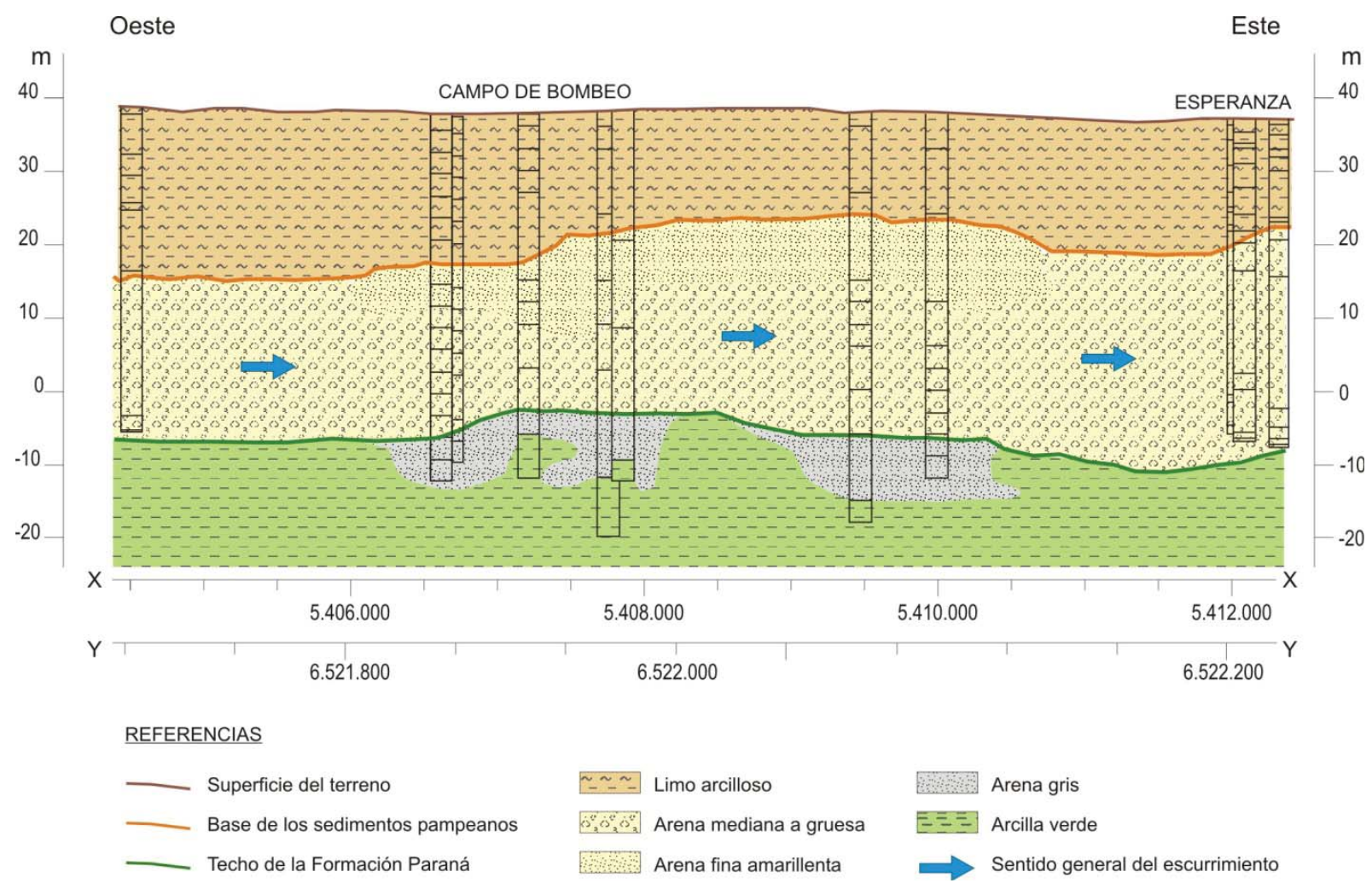

Fig. 2. Modelo conceptual del sistema acuífero

do de buen rendimiento y calidad (espesor: 25-35 m; T=600 m2/día), desde donde se extrae el agua para el abastecimiento de la población. Subyaciendo a ellas, la Formación Paraná (Mioceno marino): porción cuspidal, arenas grises, arcillas arenosas y arcillas verdes. Agua de elevada salinidad; a una profundidad entre 45 y $53 \mathrm{~m}$ (Paris, Tujchneider, Pérez, D’Elía, 2014). Para (Tujchenider, Pérez, Paris, D'Elía, 2005), el comportamiento hidráulico de este sistema es de tipo multicapa, con la posibilidad de flujo descendente y/o ascendente a través del acuitardo, en función de las relaciones de carga hidráulica imperantes. La extracción de agua del acuífero semiconfinado puede inducir no sólo el flujo desde el acuífero libre suprayacente, incrementar la afluencia lateral de agua del propio acuífero e inducir el ascenso de agua salada. La recarga local al primer nivel acuífero está en el orden de los $63 \mathrm{~mm} / \mathrm{año}$ y al segundo nivel, a través del acuitardo es de aproximadamente $18 \mathrm{~mm}$ (D'Elía, Tujchneider, Paris, Pérez, 2013). El análisis de $\partial^{18} \mathrm{O}$ y $\partial^{2} \mathrm{H}$ en el agua subterránea en relación a la recta meteórica muestra que la recarga proviene de la precipitación local, que ocurre en los meses de verano y otoño y no refleja procesos de evaporación significativos. Asimismo, los valores de tritio detectados indican que el tiempo de residencia del agua en el acuífero semiconfinado es mayor a 50 años y que en el acuífero libre resulta de una mezcla entre recarga submoderna y reciente. Particularmente, los contenidos de tritio detectados en uno de los pozos de monitoreo del acuífero semiconfinado sugieren la mezcla de agua de los dos niveles acuíferos. La presencia de nitrato estaría corroborando esta situación, favorecida además por la discontinuidad del 
manto acuitardo. La zona de emplazamiento del campo de bombeo fue categorizada como un área de alto potencial de recarga (D’Elía, Tujchneider, Paris, Pérez, 2013).

El sentido general de escurrimiento subterráneo es de Oeste a Este con un gradiente hidráulico promedio de $2 * 10^{-3}$. A partir de 20 años de registros de caudal en distintas secciones del río Salado (ubicado al Este del área del estudio) una descarga anual del sistema del orden de los $55 \mathrm{Mm}^{3}$.

Las aguas son de tipo bicarbonatado sódico. Sin embargo, desde mediados de la década del '90, en algunos pozos de la zona urbana se evidenciaron variaciones que derivaron en el cambio de tipo de agua bicarbonatada hacia el tipo clorurada sódica. En particular, en la ciudad de Esperanza, durante el período '94-'96 se pudo comprobar el efecto que la mayor extracción produjo no sólo en la dirección del escurrimiento, sino también en la salinidad del agua (expresada en términos de conductividad eléctrica). Incluso fueron identificas las áreas afectadas por el ascenso de agua salada utilizado análisis de agrupamiento jerárquico (Tujchenider, Pérez, Paris, D'Elía, 2005) y otros métodos estadísticos multivariados que, con distinto fundamento matemático permiten realizar la validación cruzada de los resultados.
A partir de una base de datos que integra 437 registros químicos provenientes de diversas fuentes: organismos provinciales, empresas prestatarias del servicio, universidad, etc. y cubre un período de observación entre 1990 y 2007, donde se suscitaron cambios tanto en el manejo como en la gobernanza del agua, se definieron utilizando técnicas estadísticas multivariadas (agrupamiento jerárquico y por K-medias y componentes principales) para definir indicadores de calidad del agua (Paris, Tujchneider, Pérez, D’Elía, 2014) (Tabla 1). Estos indicadores presentados deberían monitorearse para garantizar la gestión sostenible del sistema acuífero, evitando la ruptura del equilibrio hidráulico entre el acuífero puelche (semiconfinado, donde se extrae el agua para consumo humano), el pampeano (libre) y el agua salada alojada en la Formación Paraná (de origen marino). Respecto de la CE (Conductividad Eléctrica del agua), no puede dejar de reconocerse su practicidad de medición en campo y en laboratorio, y que verdaderamente puede ofrecer indicios de salinización. Sin embargo, en este estudio se ha mostrado que no es un parámetro que ofrece una alerta temprana de manifestación del proceso de salinización del acuífero en explotación por ascenso de agua salada subyacente, ni tampoco del aporte desde el acuífero libre superior.

Tabla 1. Indicadores de calidad del agua de abastecimiento.

\begin{tabular}{cccccccc} 
& $\mathbf{C E}$ & $\mathbf{R S}^{(*)}$ & $\mathbf{C l}^{-}$ & $\mathbf{S O}_{\mathbf{4}}{ }^{2-}$ & $\mathbf{N O}_{3}{ }^{-}$ & $\mathbf{C a}^{2+}$ \\
\hline & & & & & & & \\
& $\boldsymbol{\mu} \mathbf{S} / \mathbf{c m}$ & $\mathrm{mg} / \mathrm{L}$ & $\mathrm{mg} / \mathrm{L}$ & $\mathrm{mg} / \mathrm{L}$ & $\mathrm{mg} / \mathrm{L}$ & $\mathrm{mg} / \mathrm{L}$ \\
\hline Umbral & 1368 & 2062 & 68 & 100 & 8 & 12 \\
\hline Alerta & 1541 & 2335 & 109 & 135 & 42 & 30 \\
\hline
\end{tabular}

(*) Los valores de RS fueron estimados como en función de la función de regresión lineal entre los datos disponibles $(\mathrm{RS}=1.5853 * \mathrm{CE}-106.97)$. 
A pesar de la importancia que reviste el agua subterránea, hasta la fecha ningún esquema de protección ha sido formalmente definido y llevado adelante, considerando la intensa actividad agrícola-ganadera e industrial que se desarrolla en la región.

Las inspecciones sanitarias de las obras de captación llevadas a cabo siguiendo una adaptación de la metodología estandarizada por Lloyd y Helmer -en (Foster, Hirata, Gomes, D'Elía, Paris, 2003) -, en un área de $200 \mathrm{~m}$ alrededor de los pozos, mostraron que los pozos revisten una categoría de contaminación entre moderada y muy alta (Tabla 2) (Paris, D'Elía, Pérez, Pacini, 2017). Las inspecciones sanitaras se realizan en las propias instalaciones de bombeo, son directas y visuales. Se basan en la puntuación de una serie de factores que condicionan el peligro de contaminación de las fuentes de suministro. En general se contemplan una serie de preguntas cuya respuesta es SI/NO para vincular afirmativamente a la presencia de una amenaza de contaminación (Schmoll, Howard, Chilton, Chorus, 2006). El protocolo o formulario a utilizar en las tareas de inspección en campo deberá ser diseñado y validado especialmente para cada caso en función de las características del sistema sanitario de cada localidad, su ubicación (área urbana o rural) y condiciones ambientales (características del sistema natural y del medio social). Normalmente las tareas de inspección en campo se realizan en un área de 200 a $500 \mathrm{~m}$ de radio y pueden complementarse con interpretación de imágenes satelitales y fotografías aéreas.

Tabla 2. Categorización del peligro de contaminación de los pozos de explotación

\begin{tabular}{|l|c|c|c|}
\hline \multicolumn{1}{|c|}{ Categoría } & \multicolumn{1}{|c|}{ Muy Alto } & Alto & \multicolumn{1}{c|}{ Moderado } \\
\hline Pozos & $\begin{array}{l}\mathrm{r} 0, \mathrm{r} 1, \mathrm{r} 2, \mathrm{r} 17, \mathrm{r} 18, \mathrm{r} 19, \\
\mathrm{r} 22, \mathrm{r} 24, \mathrm{r} 25, \mathrm{r} 34, \mathrm{e} 18\end{array}$ & $\begin{array}{l}\mathrm{r} 3, \mathrm{r} 6, \mathrm{r} 11, \mathrm{r} 15, \mathrm{r} 16, \mathrm{r} 20, \\
\mathrm{r} 21, \mathrm{r} 23, \mathrm{r} 30, \mathrm{e} 8\end{array}$ & $\begin{array}{l}\mathrm{r} 4, \mathrm{r} 5, \mathrm{r} 7, \mathrm{r} 8, \mathrm{r} 9, \mathrm{r} 10, \\
\mathrm{r} 12, \mathrm{r} 13, \mathrm{r} 29, \mathrm{e} 14\end{array}$ \\
\hline $\begin{array}{l}\text { Cantidad de Pozos por } \\
\text { categoría }\end{array}$ & 11 & 10 & 10 \\
\hline
\end{tabular}

Asimismo, se delimitaron perímetros de protección con los siguientes métodos $(\mathrm{Pa}-$ ris, D'Elía, Pérez, Pacini, 2017):

- Radio fijo arbitrario, para el área operacional del pozo (10 $\mathrm{m}$ de radio).

- Radio fijo calculado en función del tiempo y Wyssling, para el área de protección microbiológica y área de vigilancia. Lo cual dio como resultado áreas de $70 \mathrm{~m}$ de radio y $100 \mathrm{~m}$, respectivamente. En estas áreas se realizó la categorización de la amenaza de contaminación de las actividades que se desarrollan en el terreno por el método POSH (Foster, Hirata, Gomes, D'Elía, Paris, 2003), resultado en algunos casos moderada y en otros reducida.

Se ha concluido en que la mayor atención debe ponerse en el área operacional del pozo, especialmente en los 11 pozos catalogados con un muy alto peligro de contaminación. En estos casos es indispensable que se lleven a cabo acciones inmediatas para controlar y revertir esta situación, garantizar la seguridad hídrica de Esperanza y Rafaela. Esto requiere: 
- Restringir y controlar las actividades que se desarrollan en el área operacional del pozo de explotación, para evitar que las cargas contaminantes que se encuentran en la superficie entre en contacto con el agua subterránea en el área de bombeo.

- Señalizar la totalidad de los pozos de explotación. Esta medida, tan simple es fundamental para que la población sepa y valore que de esa obra depende el suministro de agua.

- Adecuar el área de operación en todos los pozos para brindarle al operador mejores condiciones laborales. Las dimensiones mínimas deberán ser $10 \mathrm{~m}$ por $10 \mathrm{~m}$ o $10 \mathrm{~m}$ de radio. Deberán contar con piso de concreto, disponer iluminación y tener un mantenimiento adecuado (limpieza y desmalezado). Este es el primer PPP, el área operacional del pozo.

- Instalar un cerco perimetral en todos los pozos de explotación para su correcta protección contra el vandalismo y accidentes.

- Colocar generadores eléctricos en todos los pozos para asegurar la continuidad del servicio de agua potable.

- Instalar en todos los pozos caudalígrafos o en su defecto caudalímetros con un protocolo de lectura riguroso, para medir y controlar el caudal de agua extraído. Es necesario contar con mediciones reales del caudal para no tener que recurrir a estimaciones y promedios.

- Realizar el mantenimiento y limpieza periódica del pozo de explotación (control del estado de las bombas, cañerías, caudalímetros, entre otros).
- En aquellos pozos ubicados a la vera de caminos, revestir el canal cuneta, como mínimo en las inmediaciones del pozo de explotación, al menos $20 \mathrm{~m}$ a cada lado del pozo.

- Re establecer las estaciones o pozos de monitoreo para realizar un control sistemático del estado del acuífero tanto en términos de la cantidad (niveles) como de la calidad del agua subterránea.

- Realizar los acuerdos institucionales necesarios para garantizar un fácil acceso a las obras de captación (mantenimiento de caminos comunales, desmalezamiento, etc.).

En el caso del campo de bombeo de Esperanza, el $45 \%$ de los pozos se localiza en terrenos propiedad de la empresa y el $55 \%$ restante en la vía pública. Se recomienda realizar acuerdos institucionales $\mathrm{y} / \mathrm{u} \mathrm{im}$ plementar los instrumentos de gestión más apropiados (legales como restricciones al dominio y/o servidumbres, económicos como multas e indemizaciones, educativos, informativos, de concientización, etc.) con los gobiernos municipal y/o provincial para evitar se incremente esta amenaza de contaminación. Un cambio en el uso del territorio (por ejemplo, de agrícola a industrial), de manejo (riego complementario, rotación de cultivos, intensificación en el uso de agroquímicos, etc.), cambio en el tipo de actividad industrial o en la infraestructura o jerarquía de un camino o ruta, etc., provocaría un aumento de la amenaza de contaminación a la que se exponen las obras de captación de agua subterránea. Además, debe tenerse en cuenta que estos valores resultan de la consideración de un caudal de explotación promedio del orden de $60-70 \mathrm{~m}^{3} / \mathrm{h}$. Esta tasa de extracción debe ser regulada y manteni- 
da, pues no sólo condiciona la validez de las zonas definidas, sino que cuando se sobrepasa induce la afluencia de agua de diferente calidad hacia el cuerpo acuífero en explotación como se presentó en este trabajo. Por ello, es fundamental contar con mediciones sistemáticas de la profundidad del nivel de agua que permitan cuantificar las relaciones hidráulicas de los distintos cuerpos acuíferos a través de los diferenciales de energía que se producen como consecuencia de la explotación, aportando otros elementos de juicio a la interpretación de la información hidroquímica realizada.

\section{Caso de estudio: Interacción agua subterrá- nea-superficial en la ciudad de Santa Fe}

La interacción entre el agua superficial y la subterránea suele ser compleja, más aún cuando se sustancia en un ambiente urbano. La escala de trabajo, los factores que intervienen, la dinámica de los procesos que se producen y los intereses del sistema social relacionado son relevantes para comprender esta interacción. En el caso de la ciudad de Santa $\mathrm{Fe}$, esto cobra especial importancia por su ubicación a orillas de dos importantes cursos de agua (el río Salado y de la laguna Setúbal -alimentada por el río Paraná). Las inundaciones son recurrentes y el efecto de la variabilidad climática en los eventos hidrometeorológicos cada vez es más acentuado, dando lugar a una alternancia de períodos húmedos - eventos lluviosos muy intensos y geográficamente concentrados $\mathrm{y}$, en contraposición, períodos más secos más prolongados. Por otra parte, el proceso de urbanización se produce hacia áreas que no cuentan con red de alcantarillado cloacal y/o se abastecen por perforaciones domiciliarias y además, contempla el uso del espacio subterráneo para el tendido de redes de servicio, sótanos, estacionamientos, fundaciones de las construcciones en altura, etc. Esto lleva a que la hidrodinámica del sistema en su conjunto deba ser considerada para definir las mejores alternativas tecnológicas de la infraestructura urbana.

A partir del año 2008 se ha comenzado a trabajar para lograr una caracterización hidrogeológica ambiental del sistema acuífero que subyace a la ciudad de Santa Fe, y evaluar su respuesta ante distintos escenarios hidrometeorólogicos extremos.

Santa Fe es la capital política y administrativa de la provincia del mismo nombre. Está situada en la región centro-este del país y tiene una población de aproximadamente 400.000 habitantes. Es un foco comercial, industrial, financiero, académico, profesional y productivo muy importante de la región. El crecimiento urbano se ha materializado hacia el norte, suroeste y oeste de la ciudad. Los sectores suroeste y oeste son considerados de alto riesgo hídrico pluvial y fluvial, no solo por la amenaza hidrológica sino por la vulnerabilidad del sistema social que en ella habita (asentamientos espontáneos de familias de escasos recursos económicos). En el sector norte, los asentamientos se produjeron en áreas suburbanas parceladas convencionalmente que luego se constituyeron en urbanizaciones formales y en su mayoría carecen de servicios sanitarios de agua potable y cloacas. En estos asentamientos el abastecimiento de agua se realiza a través de perforaciones comunitarias y/o perforaciones domiciliarias y el saneamiento a través de sistemas de pozos sépticos. Estos asentamientos conviven con áreas hortícolas, ladrilleras, cavas (depresiones topográficas) y con el antiguo relleno sanitario de la ciudad, que operó durante el período 
1996-2010. Si bien en este sector los niveles topográficos son elevados, los problemas de anegamientos por lluvias son recurrentes (Fornari, D'Elía, Fedele, 2013).

En este contexto, y con el objeto de jerarquizar el área norte de la ciudad, el Gobierno Municipal de la ciudad de Santa Fe ha encarado el desarrollo e implementación del denominando Plan Norte. Dicho plan comenzó a ejecutarse en 2016 dando lugar a una intervención integral de toda la zona Norte de la Ciudad de Santa Fe que abarcan entre otras, obras para mejorar los servicios públicos, la comunicación y la creación de sectores recreativo-cultural.

Las características geológicas locales se conocen a través de perfiles litológicos de perforaciones realizadas en el área con fines de abastecimiento público en la década de 1990 y de 23 pozos de monitoreo realizados con fines de investigación, en el año 2008 y 2009 en el marco de las tareas de investigación de la FICH-UNL. La profundidad de las perforaciones de abastecimiento público varía entre 25 y $40 \mathrm{~m}$ y entre 6 y $15 \mathrm{~m}$ la profundidad de los pozos de monitoreo con un tramo filtrante de 1,5 a 2 m (Figura 3). Las muestras de subsuelo obtenidas en estos sondeos fueron analizadas en el Laboratorio de Sedimentología de la FICHUNL y con ellas se elaboraron los respectivos perfiles litológicos. La interpretación de esta información permitió elaborar la correlación litoestratigráfica que se presenta en la Figura 4 para la sección A-A', ubicada en el extremo norte de la ciudad (D’Elía, Paris, Tujchneider, Pérez, Pagliano, Gualini, 2011).

La columna hidrogeológica local comprende: un basamento acuícludo formado por arenas grises y arcillas verdes de la Formación Paraná, superiormente, se encuen- tran arenas y arenas gravosas de $30 \mathrm{~m}$ de espesor promedio que pertenecen a la Formación Ituzaingó (conocida como arenas "puelches"). Sobre ellas y hasta la superficie, se apoyan sedimentos cuaternarios de granulometría más fina (arenas finas a limos, con arcillas) de origen eólico, de aproximadamente $6 \mathrm{~m}$ de espesor promedio. Esta secuencia sedimentaria da lugar a un acuífero multicapa que estaría comportándose como libre con drenaje diferido. La recarga de este acuífero es principalmente directa proveniente de las precipitaciones y eventualmente de los cuerpos de agua superficial con los que se encuentra hidráulicamente conectado, mientras que la descarga se produce en el río Salado al O y la Laguna Setúbal al E (D'Elía, Paris, Tujchneider, Pérez, Pagliano, Gualini, 2011).

Desde agosto de 2008, en los pozos de monitoreo se vienen realizando mediciones mensuales de niveles de agua subterránea y automáticas a paso diario en uno de los pozos (P1) donde se encuentra instalado un freatígrafo a presión Global Water GW16. El agua precipitada tarda algunos pocos días en alcanzar el acuífero (Figura 5). El comportamiento de los niveles piezométricos resulta similar en todos los pozos de monitoreo. En el sector oeste el nivel freático es superior al nivel del río Salado indicando que el río es efluente del acuífero. Sin embargo, durante los meses de noviembre y diciembre de 2009 la relación se invierte, con lo cual se vuelve influente. En el sector este los niveles observados superan a los niveles de la Laguna Setúbal durante el período diciembre 2009 a marzo de 2013, indicando el carácter efluente de este cuerpo de agua superficial. Las Figura 6 y 7 muestran una porción de los registros disponibles para el pozo de monitoreo P8 y P4, ubicados en el 
O y E, respectivamente (D'Elía, Paris, Tujchneider, Pérez, Pagliano, Gualini, 2011)
(Fornari, D’Elía, Fedele, 2013) (Pagliano, D’Elía, 2013).

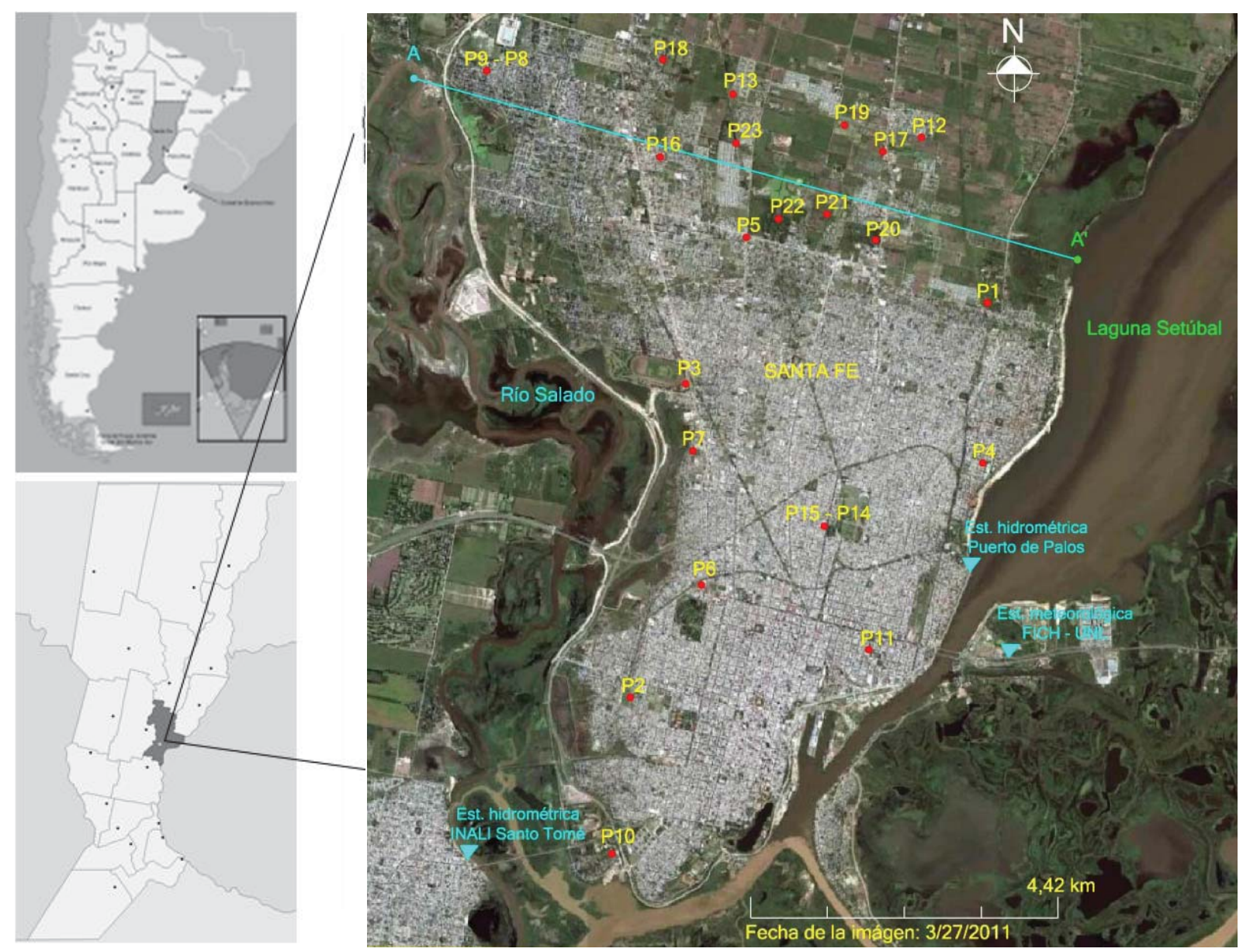

Fig. 3. Ubicación del área de estudio y de las perforaciones de estudio.

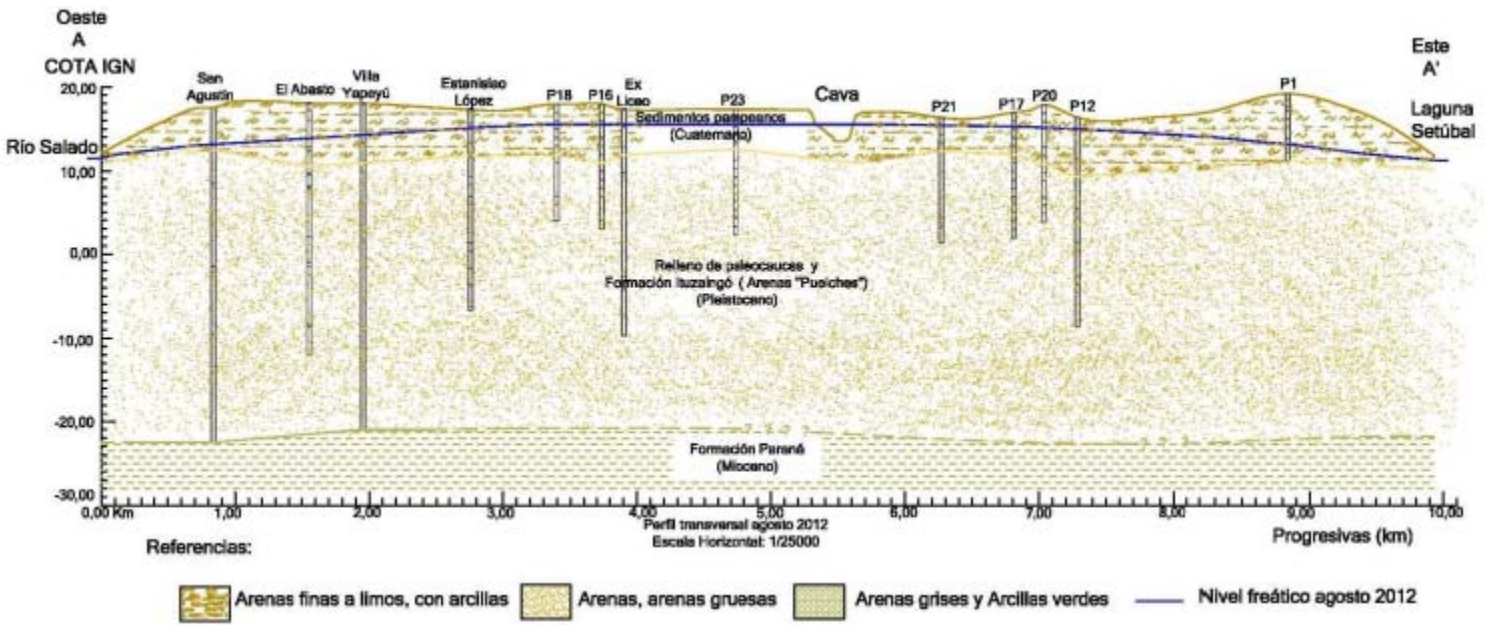

Fig. 4. Correlación litoestratigráfica oeste-este en el sector norte de la ciudad. 


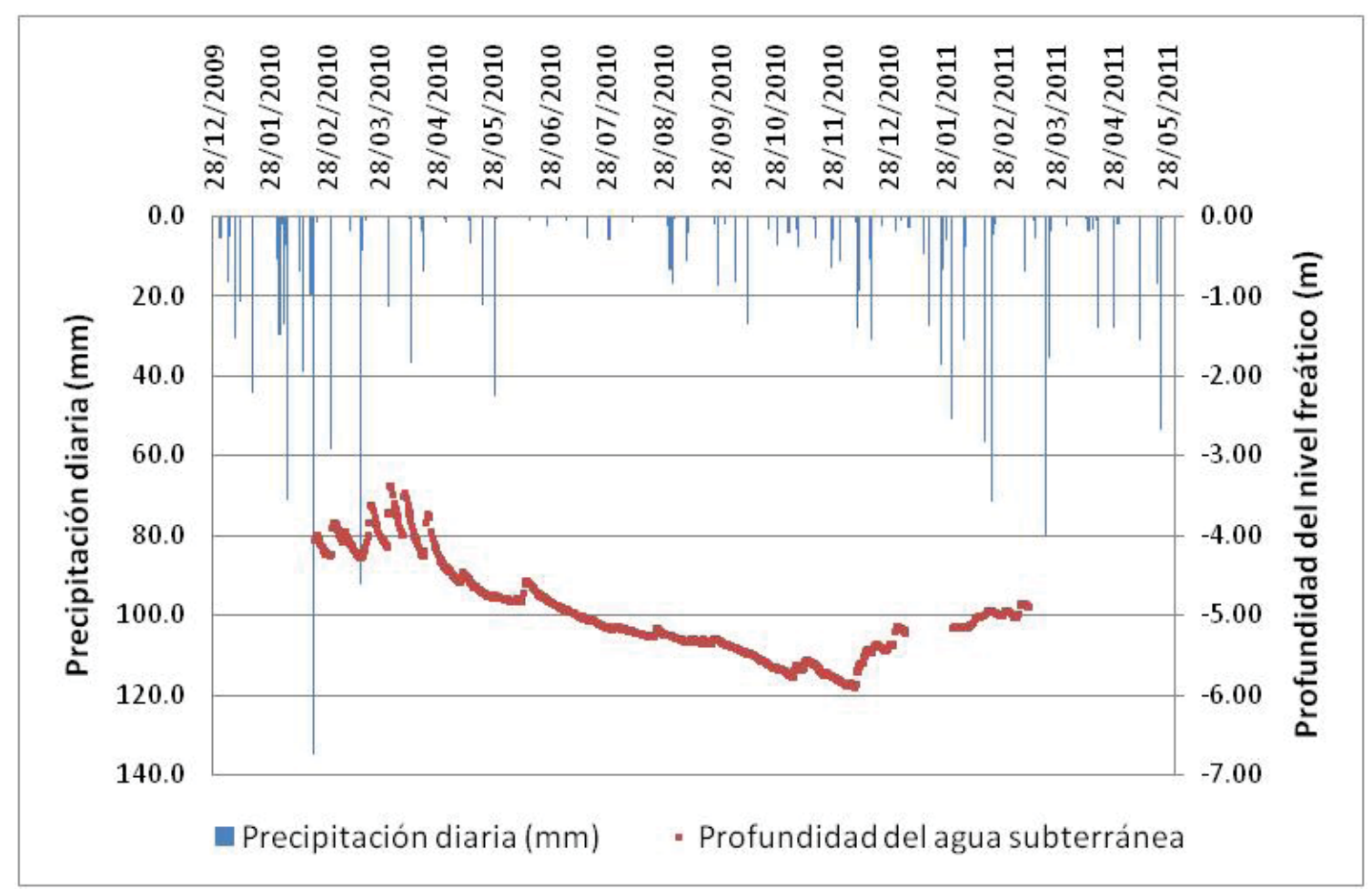

Fig. 5. Variación de la profundidad del nivel freático y la precipitación diaria.

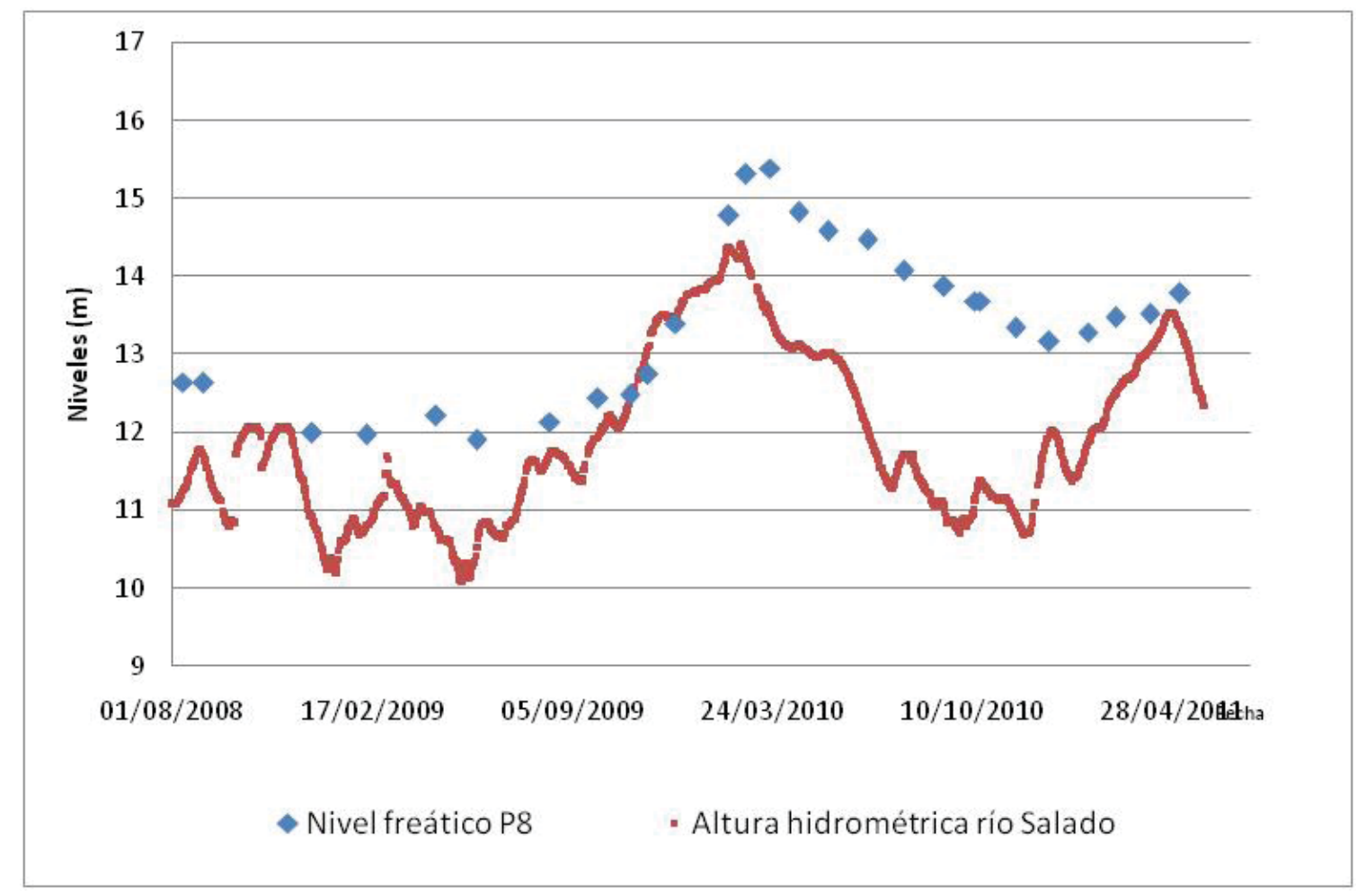

Fig. 6. Variaciones del nivel freático y del río Salado. 


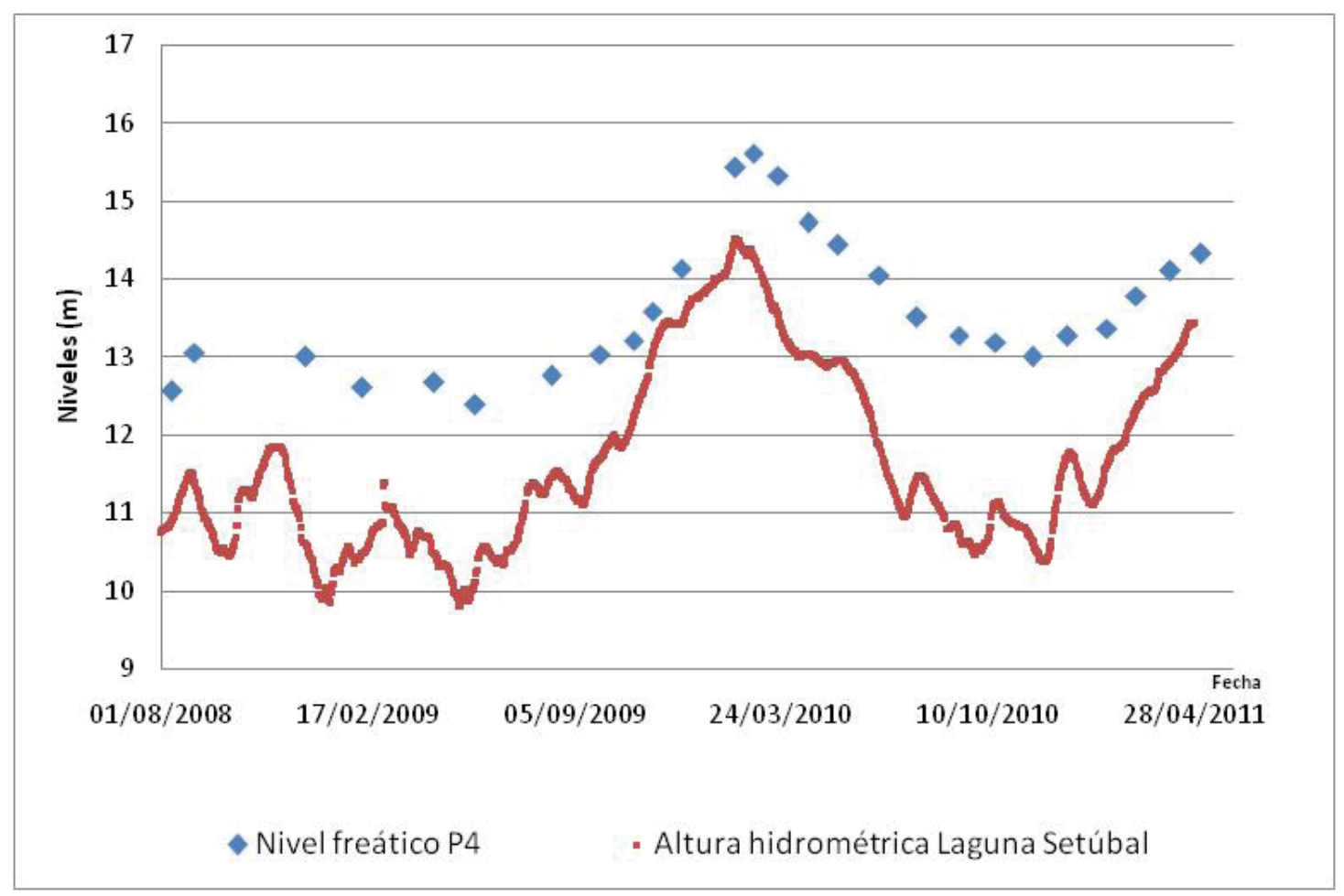

Fig. 7. Variaciones del nivel freático y de la Laguna Setúbal.

La zona de recarga del acuífero se encontraría en el norte de la ciudad donde se registran los mayores niveles freáticos (mayores a $15 \mathrm{~m})$, la dirección del escurrimiento subterráneo es hacia el sur, oeste y este, donde se encuentran los cuerpos de agua superficiales que son considerados zonas de descarga (a un nivel del orden de $11 \mathrm{~m}$ ). Sin embargo, esta situación podría invertirse en épocas de crecidas de los ríos Salado y/o Paraná, como se mencionó anteriormente. Las curvas isofreáticas correspondientes a setiembre 2010 se presentan en la Figura 8.

Se determinó la vulnerabilidad del acuífero sobre la base de las características litológicas de la zona no saturada, el tipo de acuífero y la profundidad del agua subterránea, mediante el método GOD (Foster, Hirata, Gomes, D'Elía, Paris, 2003). Es posible afirmar que la vulnerabilidad a la contaminación del acuífero resulta entre media y alta.
Se realizan trimestralmente determinaciones in situ de $\mathrm{pH}, \mathrm{CE}$, temperatura $\mathrm{y}$ anualmente se toman muestras de agua para análisis físico-químicos en los pozos de monitoreo. Se realizan determinaciones de $\mathrm{HCO}_{3}^{-}, \mathrm{SO}_{4}=\mathrm{Cl}^{-}, \mathrm{NO}_{3}^{-}, \mathrm{Na}^{++}, \mathrm{K}^{+}, \mathrm{Ca}^{++}, \mathrm{Mg}$ ${ }^{++}, \mathrm{Fe}^{++}, \mathrm{Mn}^{++}$, alcalinidad y residuo seco según las técnicas indicadas en el Standart Methods for Examination of Water and Wastewater, 19Ed. Según la clasificación de Piper-Hill, el un 60\% de las muestras corresponden al tipo bicarbonatada cálcica-magnésica, el 23\% son bicarbonatadas sódicas y el resto clorurada y/o sulfatada sódica.

En 2009 se realizaron determinaciones de ${ }^{18} \mathrm{O},{ }^{2} \mathrm{H}$ y tritio de las muestras de agua subterránea en el Instituto de Geología y Geocronología Isotópica (INGEIS-CONICET). Los valores de $\delta^{18} \mathrm{O}$ y $\delta^{2} \mathrm{H}$ del agua subterránea en el área de estudio varían entre $-4,0$ y $-7,9 \%$ y entre $-22,0$ y $-47,0 \%$, res- 
pectivamente. Estos valores no difieren significativamente del contenido medio anual ponderado de la precipitación en el área de estudio, lo que corroboraría su origen meteórico. Además, teniendo en cuenta su ubicación respecto de la línea de agua meteórica local, no habrían tenido lugar procesos significativos de evaporación durante el proceso de infiltración hacia el ambiente subterráneo. El contenido de tritio de las muestras de agua subterránea varía entre 1,7 y 3,0 UT, con lo cual se deduce, a partir de una interpretación cualitativa de estos resultados, que se trata de una mezcla entre aguas submodernas (aquellas que han sido recargadas en las últimas décadas y por lo tanto forman parte del ciclo hidrológico activo) y aguas de recarga reciente (D'Elía, Paris, Tujchneider, Pérez, Pagliano, Gualini, 2011).

En la zona norte de la ciudad, en atención al plan de desarrollo municipal, se realizó el inventario de actividades potencialmente contaminantes al subsuelo (APC) y se clasificaron según la amenaza de contaminación al agua subterránea por el método POSH (Foster, Hirata, Gomes, D'Elía, Paris, 2003). Considerando la vulnerabilidad del acuífero entre media y alta, se definieron áreas niveles de acción prioritarios para el control de la contaminación. La Tabla 3 sistematiza los resultados logrados (Vera, D'Elía, 2017).
Se realizó también la caracterización hidrológica ambiental de la "cava Borgo", depresión topográfica ubicada en el sector norte, de especial importancia en el plan de desarrollo municipal. Se realizó una batimetría en 2012 donde se determinó que la cava tenía una profundidad máxima de $2,5 \mathrm{~m}$ (con una capacidad de almacenamiento máxima de $0,32 \mathrm{Hm}^{3}$. Esto indicaría que el lecho de la misma se encuentra en los sedimentos cuaternarios de la columna hidrogeológica local. Este sector podría considerarse como una de las áreas de recarga del acuífero, existiendo una vinculación hidráulica entre la cava y el sistema acuífero (Fornari, D'Elía, Fedele, 2013). En la misma porción norte se realizó la modelación del flujo subterráneo con Visual Modflow y Modpath, para simular la trayectoria del transporte advectivo de contaminantes evaluar el destino final del lixiviado del antiguo relleno sanitario. Los resultados indican que, en épocas lluviosas, cuando los niveles del agua subterránea están próximos a la superficie, existen relaciones entre el agua subterránea, los residuos sólidos urbanos y los lixiviados producidos por el relleno sanitario; que la circulación del agua subterránea es desde el centro del área de estudio hacia los cuerpos de agua superficiales; que un contaminante aplicado en el sitio del relleno sanitario viajaría hacia la Laguna Setúbal (Pagliano, D'Elía, 2016). 


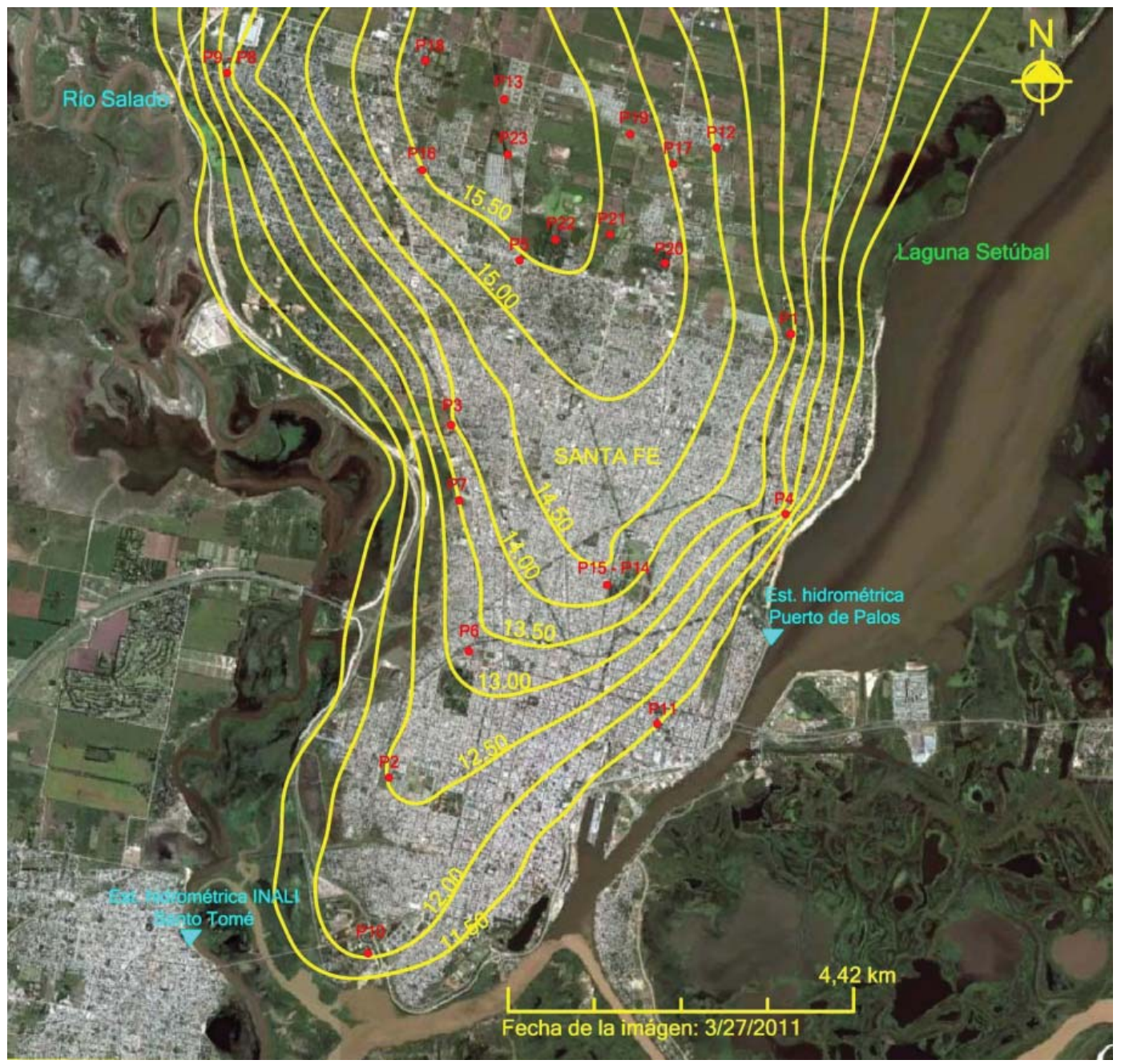

Fig. 8. Curvas isofreáticas, setiembre de 2010.

Tabla 3. Fuentes potenciales de contaminación y nivel de priorización de acciones.

\begin{tabular}{|c|c|c|c|c|}
\hline Actividad & Tipo & Categoría POSH & Cantidad & $\begin{array}{c}\text { Nivel de } \\
\text { priorización de } \\
\text { acciones }\end{array}$ \\
\hline Industria Tipo 1 & Puntual & Reducido & 2 & 2 \\
\hline Industria Tipo 3 & Puntual & Elevado & 18 & 1 \\
\hline Estación de Servicio & Puntual & Moderado & 2 & 1 \\
\hline $\begin{array}{c}\text { Disposición de residuos } \\
\text { sólidos }\end{array}$ & Puntual & Elevado & 1 & 1 \\
\hline Cementerio & Puntual & Reducido & 1 & 1 \\
\hline Saneamiento in situ & Difusa & Elevado & 1400 ha & 1 \\
\hline Cultivo de hortalizas & Difusa & Elevado & 217 ha & \\
\hline
\end{tabular}




\section{CONSIDERACIONES FINALES}

Los avances logrados en el conocimiento de las dos áreas urbanas señalan la importancia de la Hidrología Subterránea. Así la ciencia se transforma en un motor que genera soluciones efectivas para que la sociedad alcance las metas del desarrollo sostenible y la seguridad hídrica.

La construcción de sólidos esquemas de gobernanza sólo será posible a partir de la democratización del conocimiento. Esto permite salvar asimetrías, garantizando la participación y compromiso de los ciudadanos, habilitando la definición de políticas, la sostenibilidad de la planificación y el empleo estratégico, eficaz y eficiente de instrumentos de gestión. Todo ello facilita el clima de paz y gobernabilidad de los recursos de la comunidad, entre ellos los hídricos.

\section{REFERENCIAS BIBLIOGRÁFICAS}

D'Elia, M., Paris, M., Tujchneider, O., Pérez, M., Pagliano, M., Gualini, S. (2011). Aguas subterráneas en áreas urbanas. En: Actas VII Congreso Argentino de Hidrogeología y IV Seminario HispanoLatinoamericano sobre Temas Actuales de la Hidrología Subterránea. Captación y Modelación de Agua Subterránea. 4653. Salta, Argentina: Universidad Nacional de Salta.

D'Elia, M., Tujchneider, O., Paris, M., Pérez, M. (2013). Groundwater recharge quantification for the sustainability of ecosystems in plains of Argentina. En: Groundwater and Ecosystems. L. Ribeiro, T. Stiger, A. Condeso de Melo, A. Medeiros, \& T. \&. Group. Ed. IAH Selected Papers on Hydrogeology 18: 137-151. Leiden, Paises Bajos: CRC Press/Balkema.
Filí, M., Tujchneider, O., Paris, M., Pérez, M., D'Elia, M. (1999). Estudio del sistema de aguas subterráneas en el área de Esperanza-Humboltd y zona de influencia. Servicio Especializado de Asistencia Técnica. Convenio Aguas Provinciales de Santa Fe y la Universidad Nacional del Litoral. Informe Final. Universidad Nacional del Litoral, Santa Fe. 214p.

Fornari, E., D'Elia, M., Fedele, A. (2013). Relaciones Hidráulicas entre un sistema acuífero y cuerpos de agua superficial en áreas urbanas. En: Anales XXIV Congreso Nacional del Agua: 1-2. San Juan, Argentina.

Foster, S., Hirata, R., Gomes, D., D'Elia, M., Paris, M. (2003). Protección de la Calidad del Agua Subterránea. Guía para empresas de agua, autoridades municipales, y agencias ambientales. (G. m. Bank., Ed.) Madrid, España: Mundiprensa. $153 \mathrm{p}$

Pagliano, M., D'Elia, M. (2016). Evaluación de las relaciones hidraúlicas e hidroquímicas entre un relleno sanitario y un sistema acuífero en un área de llanura. En: Actas XIII Congreso Argentino de Hidrogeología y V Seminario HispanoLatinoamericano sobre Temas Actuales de la Hidrología Subterránea: 1-8. Catamarca, Argentina.

Paris, M., D’Elia, M., Pérez, M., Pacini, J. (2017). Wellhead protection zone for sustainable ground water supply. Sustainable Water Resources Management Journal. ISSN: 2363-5045 (electronic version). Springer, Alemania, Berlín. DOI: 10.1007/s40899-017-0156-x

Paris, M., Tujchneider, O., Pérez, M., D'Elia, M. (2014). Protección de pozos de abastecimiento. Indicadores de la calidad del agua subterránea. Revista 
Ciencia y Tecnología del Agua. Instituto Mexicano de Tecnología del Agua (IMTA), V (4): 1-12. ISSN 2007-2422. https://www.imta.gob.mx/tyca/revistadigital198237645imta_comunicacion/ revistas-2014/rev-04-2014/files/assets/ basic-html/index.html\#7

Peña, H. (2016). CEPAL-Comisión Económica para América Latina y el Caribe. (N. Unidas, Ed.) Recuperado el 2017, de http://repositorio.cepal. org/bitstream/handle/11362/40074/1/ S1600566_es.pdf

PNUD. (2015). Programa de Naciones Unidas para el Desarrollo. Recuperado el 2015, de http://www.undp.org/content/ undp/es/home/sustainable-developmentgoals.html

Shah, T. (2016). Global Water Partnership. Recuperado el 2017, de http://www.gwp. org/globalassets/global/toolbox/publications/background-papers/tec22_espanol.pdf

Schmoll, O., Howard, G., Chilton, J., Chorus, I. (2006). Protecting Groundwater for Health. Managing the quality of drinking-water sources. Londres: IWA Publishing-World Health Organization. ISBN 1843390795. 155 p.

Tujchenider, O., Filí, M., Paris, M., D’Elia, M., Pérez, M. (1998). Protección de las aguas subterráneas. Caso de estudio: Ciudad de Esperanza (República Argentina). Primera Fase: Diagnóstico del sis- tema. En: Memorias IV Congreso Latinoamericano de Hidrología Subterránea. 2: 805-820. Montevideo.

Tujchneider, O., Pérez, M., Paris, M., D'Elia, M. (2005). Deterioro de fuentes de agua subterránea por ascenso de agua salada. En: Actas IV Congreso Argentino de Hidrogeología: 227-236. Río Cuarto, Córdoba.

UNESCO. (2014). Organización de las Naciones Unidas para la Educación, la Ciencia y la Cultura. Recuperado el 2017, de http://unesdoc.unesco.org/ images/0021/002180/218061s.pdf

Van Beek, E., Lincklaen Arriens, W. (2014). GWP-Global Water Partnership. Recuperado el 2017, de http://aquadoc.typepad.com/files/gwp_tec20_web.pdf

Vera, M., D'Elia, M. (2017). Definición de áreas que requieran acciones de priorización para el control de la contaminación del agua subterránea en el sector norte de la ciudad de Santa Fe (Argentina). En: Anales III Congreso Nacional de Ciencia y Tecnología Ambiental. 6p. Santa Fe. Argentina.

WWAP/ONU. (2018). UNESCO. Recuperado el 2018, de Programa de Evaluación Mundial de los Recursos Hídricos - WWAP: http://www.unesco.org/ new/es/natural-sciences/environment/ water/wwap/wwdr/2018-nature-basedsolutions/\#c1654856 